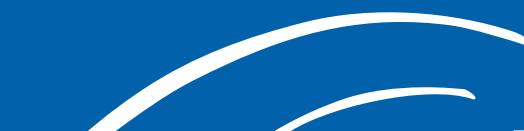

\section{Reutilización de agua para la agricultura y el medioambiente}

\author{
Water Reuse in Agriculture and the Environment
}

\author{
Amparo Melián-Navarro \\ Universidad Miguel Hernández. Orihuela, Alicante, España. amparo.melian@umh.es
}

M. a Ángeles Fernández-Zamudio

Instituto Valenciano de Investigaciones Agrarias. Moncada, Valencia, España. fernandez_marzam@gva.es

Resumen - En el presente artículo se revisan los usos que pueden tener las aguas regeneradas en agricultura y con una finalidad medioambiental. Aunque tras un correcto tratamiento de depuración las opciones de uso de las aguas residuales son muchas, se destinan mayoritariamente a una finalidad agraria. Las ventajas de regar con esta fuente complementaria de agua es que se reduce la sobreexplotación de los acuíferos y se puede aprovechar la carga nutricional del agua para disminuir la cantidad de fertilizante aportado. En las próximas décadas parece que además de en agricultura, las aguas regeneradas cada vez se utilizarán más para diferentes usos urbanos y ambientales. A nivel medioambiental, las aguas depuradas facilitan el mantenimiento de los humedales y los caudales mínimos ecológicos.

Abstract - This article analyses reused water for agricultural and environmental purposes. After proper purification treatments have been applied there are many options available for the use of the reclaimed wastewater, but most of it is used in agriculture. It is possible to reduce the overexploitation of aquifers with this additional source of water, and we can take advantage of the nutritional value of the water to reduce the amount of fertilizers used in commercial farming. In the next decades it seems that in addition to agriculture reclaimed water will be used increasingly for different urban and environmental purposes. At the environmental the use of treated water facilitates the maintenance of wetlands and minimum ecological flows.

\begin{abstract}
Palabras clave: depuración de agua, agua regenerada, agricultura de regadío, humedales, sostenibilidad ambiental Keywords: wastewater purification, purified water, irrigated agriculture, wetlands, environmental sustainability
\end{abstract}




\section{INTRODUCCIÓN ${ }^{1}$}

El agua es esencial para la vida y el bienestar de las personas. En el último siglo ha pasado de ser un recurso natural "inagotable" a ser "limitante" para el desarrollo económico y social.

La demanda del agua se ha elevado notoriamente en todas las zonas urbanas, donde la población hace un uso mucho más intensivo del agua del que hacía hace décadas. Esta fuerte demanda, en el caso de España, es con frecuencia mayor en áreas donde las precipitaciones son escasas y que tienen un balance hídrico claramente deficitario. A la gran exigencia de caudales y la escasez natural del recurso, se unen los problemas de contaminación y dificultades en los tratamientos, lo que complica la disponibilidad de agua y hace más necesaria la búsqueda de alternativas que garanticen el suministro.

La reutilización de las aguas es en sí un fenómeno real, un hecho constatado y empleado en todo el mundo y a lo largo de la historia de la humanidad. Desde la antigüedad los núcleos poblacionales siempre dieron importancia no solo a disponer de un volumen determinado de agua, sino que también se buscaba que esta tuviera la suficiente calidad. Sin embargo, hasta el siglo XIX no se puso de manifiesto la necesidad de una adecuada gestión del agua residual como medio de protección de la salud pública². A partir de la década de los 70 en la normativa europea se ha dado un papel prioritario a la depuración de las aguas, ya que toda el agua vertida a un cauce puede ser empleada aguas abajo con distintos fines.

El propio ciclo natural de las aguas supone que un mismo recurso sea utilizado de forma directa 0 indirecta por diversos usuarios, por lo que los procesos de regeneración de aguas que ya han sido utilizadas se vuelven imprescindibles.

Entre todos los usos que se dan a las aguas regeneradas, el mayoritario es el agrario (aproximadamente un setenta por ciento del total), básicamente para el riego de cultivos y pastos. Le siguen el uso medioambiental, sobre todo las funciones de recarga de acuíferos y la recuperación de humedales. Además, están los usos recreativos (especialmente campos de golf), el urbano y el industrial (principalmente en el sector textil). El agua depurada se utiliza para un fin agrario en alrededor de cincuenta países ${ }^{3}$, surte el $10 \%$ de todas las tierras de regadío del mundo, siendo esta su utilidad principal, pero en la actualidad también son cada día más numerosos sus fines medioambientales.

Precisamente por su relevancia y significación, en este artículo se va a desarrollar de forma explícita la utilidad y uso del agua depurada en las dos áreas que también se encuentran vinculadas entre ellas: la agricultura y el medio ambiente.

\section{Generalidades sobre el uso del agua Regenerada}

El uso cada vez más extendido de la reutilización del agua permite aumentar las dotaciones a la vez que se reduce la so-

1 Este trabajo ha sido financiado en parte por la Fundación Séneca-Agencia de la Ciencia y la Tecnología, con cargo al Proyecto: El papel de los mercados del agua en la gestión integrada de los recursos hídricos en las cuencas deficitarias. (Ref. 19325/ Pl/ 15).

2 Molinos et al. 2012.

3 Aquarec, 2006. breexplotación de los acuíferos. Esto es especialmente importante en países como los del área mediterránea, donde el aporte de este tipo de agua tiene claras ventajas económicas y medioambientales. Al contribuir al equilibrio entre la oferta y la demanda suponen una solución coste-eficacia correcta, un efecto que es aún más valorado en las zonas hidrológicamente deficientes ${ }^{4}$.

Numerosos autores ${ }^{5}$ apelan a la importancia de conseguir nuevos usos para las aguas regeneradas: "la reutilización de recursos obtenidos de la regeneración de aguas residuales debería considerarse irrenunciable, tanto desde el punto de vista social como ambiental y sanitario", ya que "además de optimizar el propio proceso de depuración, con la utilización de estos recursos no convencionales se consigue rebajar la presión de la demanda sobre los determinados recursos hídricos convencionales".

Las aguas regeneradas son un recurso más dentro de la gestión hídrica y aunque no puedan considerarse un recurso convencional sí tienen un papel clave en la planificación integral del agua. A pesar de ello su importancia a nivel cuantitativo es todavía muy baja, representando en torno a un tres por ciento del total de recursos hídricos disponibles. Sin embargo, la mejora de la calidad de los efluentes es fundamental para su posterior aprovechamiento, no hay que olvidar que el agua puede tener una vida casi ilimitada si es bien gestionada.

En líneas generales, el agua regenerada puede destinarse a actividades que no requieran un recurso de gran calidad, lo que permite liberar los volúmenes de mejor calidad para otros usos más exigentes ${ }^{6}$. Otro objetivo fundamental del procedimiento de regeneración de aguas es sobre todo ambiental, ya que con la depuración del agua se evita el vertido de contaminantes a cauces fluviales, acuíferos 0 al mar. De no hacerlo, los problemas se agravan, ya que las aguas residuales que no son tratadas generan efectos adversos como eutrofización, malos olores, impacto visual, contaminación directa de otras aguas, etc. ${ }^{7}$

De forma tradicional el agua regenerada ha sido siempre una garantía para los suministros del riego, sobre todo en las zonas con mayor déficit hidrológico, siendo utilizada en la agricultura desde hace siglos. En la actualidad es cada vez más frecuente el intercambio de agua destinada en su inicio a fines agrícolas para dedicarla a un uso urbano, una demanda creciente y en la que puede obtenerse un mayor valor económico y social. Es por ello que se dice que el agua regenerada ofrece beneficios tanto a usuarios urbanos como a agricultores y, por supuesto, al medio ambiente.

Con excepción del uso para alimentación humana y para el ganado, un agua que ha sido convenientemente depurada puede destinarse prácticamente a cualquier fin. Es cierto que su calidad final no siempre será igual, sino que dependerá del tratamiento al que haya sido sometida. El coste de obtención suele ser proporcional a la calidad final obtenida, lo que dependerá también de las características iniciales que tenía el agua residual de partida.

\footnotetext{
4 FAO, 2013.

5 Hernández et al. 2006.

6 Melgarejo, 2009.

7 Morugán-Coronado, 2011.
} 
Tabla 1. Resumen de algunas experiencias realizadas con aguas residuales

\begin{tabular}{|c|l|l|}
\hline Año & \multicolumn{1}{|c|}{ Lugar } & \multicolumn{1}{c|}{ Destino-Uso } \\
\hline $1912-1985$ & Golden Gate, California (USA) & Riego de jardines y abastecimiento lagos artificiales \\
\hline 1926 & Gran Cañón, Arizona (USA) & Cisternas wc, riego de parques y jardines \\
\hline 1929 & Pomona, California (USA) & Riego de parques y jardines \\
\hline 1942 & Baltimore, Maryland (USA) & Enfriamiento de metales y acero \\
\hline 1960 & Colorado (USA) & Riego de campos de golf \\
\hline 1961 & Irvine Ranch, California (USA) & En riegos, industria y cisterna wc \\
\hline 1962 & La Soukra (Túnez) & Riego de cultivos de naranjos \\
\hline 1969 & Wagga Wagga (Australia) & Riego de parques, zonas deportivas y cementerios \\
\hline 1970 & Enstra (Sudáfrica) & Procesos de fabricación del papel \\
\hline 1976 & Orange Water District, California (USA) & Recarga de acuíferos \\
\hline 1977 & Dan Project, Tel-Aviv (Israel) & Recarga de acuíferos y riego agrícola \\
\hline 1984 & Gobierno de Tokio (J apón) & Cisterna wc \\
\hline 1985 & El Paso, Texas (USA) & Recarga de acuíferos \\
\hline 1987 & Monterey, California (USA) & Riego de cultivos \\
\hline 1989 & Shoalhaven Heads (Australia) & Riego de parques y jardines, Cisterna wc \\
\hline 1989 & Gerona (España) & Riego de campos de golf \\
\hline 1999 & Willunga Basin, Adelaide (Australia) & Riego de cultivos de uvas \\
\hline
\end{tabular}

Fuente: Morugán Coronado, 2011, en base a un trabajo de Keremane, 2010.

Gracias a las mejoras tecnológicas implementadas en las últimas décadas y contando con las infraestructuras que posibiliten el intercambio real de derechos de aguas entre las partes involucradas, hoy por hoy son muchos los posibles usos del agua regenerada. Los principales y que se dan en diversas partes del mundo son8: agricultura (semilleros y cultivos), uso urbano no potable (lavaderos, sanitarios, baldeo de las calles, limpieza, jardines, parques, aire acondicionado, etc.), medioambiental (lucha contra incendios forestales, caudales ecológicos, recarga de zonas húmedas, recarga artificial de acuíferos, control de la intrusión marina, etc.), industria y construcción (refrigeración, alimentación de calderas, mezcla de materiales, limpieza de maquinaria) y otros muy diversos (acuicultura, fusión de nieve, limpieza de ganado, eliminación de polvo, etc.).

Algunas experiencias con aguas residuales ${ }^{9}$, según su principal destino, y de las que se tiene constancia desde principios del siglo pasado son los recopilados en la tabla 1.

Algunos de estos usos no están exentos de cierta controversia y tienen dificultades para su acepción social. Se insiste en los posibles problemas de salud pública (trasmisión de patógenos) y otras limitaciones atribuyéndoles un efecto negativo sobre la calidad de los cultivos. Ante el grupo de detractores, una correcta gestión, responsabilidad y un mayor conocimiento científico y técnico es lo que favorecerá una mayor implantación, una utilidad más eficiente y mejor aceptación social.

El uso de estas aguas está muy regulado y exige el cumplimiento de la normativa al respecto. En primer lugar, se nombra la Directiva del Consejo Europeo (1991/271/CEE) de mayo de

8 Asano, 1991.

9 Morugán-Coronado, 2011.
1991, que legislaba sobre el tratamiento de las aguas residuales urbanas. Esta norma fue transpuesta al ordenamiento jurídico del Estado español por el real decreto ley $11 / 95$ y real decreto 509/96, con los que se intenta dar cumplimiento a la norma legal europea, e impulsar el tratamiento correcto de las aguas residuales urbanas antes de su vertido. Más recientes son la Directiva comunitaria 2000/60/CE del 23 de octubre de 2000, denominada Directiva Marco del Agua, que recoge de manera muy amplia los aspectos que condicionan la protección y gestión de las aguas. Posteriormente el real decreto 1620/2007, de 7 de diciembre, se concentra de forma específica en los usos permitidos y criterios de calidad que se exigen en la reutilización de las aguas depuradas. En el caso del agua destinada a fines agrarios se encuentran en el nivel segundo de exigencias en calidad, medida en parámetros como turbidez, concentración máxima de Echerichia coli, etc., y solo por debajo de las aguas destinadas al uso urbano, donde los criterios son más exigentes.

La necesidad hace que el mayor número de sistemas o plantas depuradoras (Ilamadas también Estaciones de Depuración de Aguas Residuales, o EDAR) en nuestro país se hallen en el arco mediterráneo, Andalucía y los archipiélagos de Baleares y Canarias. En estas zonas coexiste una alta demanda urbana y/o agrícola con el agotamiento de las fuentes tradicionales de obtención de agua, debido a la salinización de los acuíferos o las escasas precipitaciones. Madrid, por su importante población, también requiere de un extenso servicio en plantas depuradoras.

En el gráfico 1 se representa el volumen de agua que se reutiliza en cada demarcación hidrográfica, destacando sobre todas las demás la demarcación del Júcar y del Segura, así como otras áreas mediterráneas. Es evidente que ante la falta de disponibilidad de agua solo cabe utilizar fuentes alternativas de suministro. 


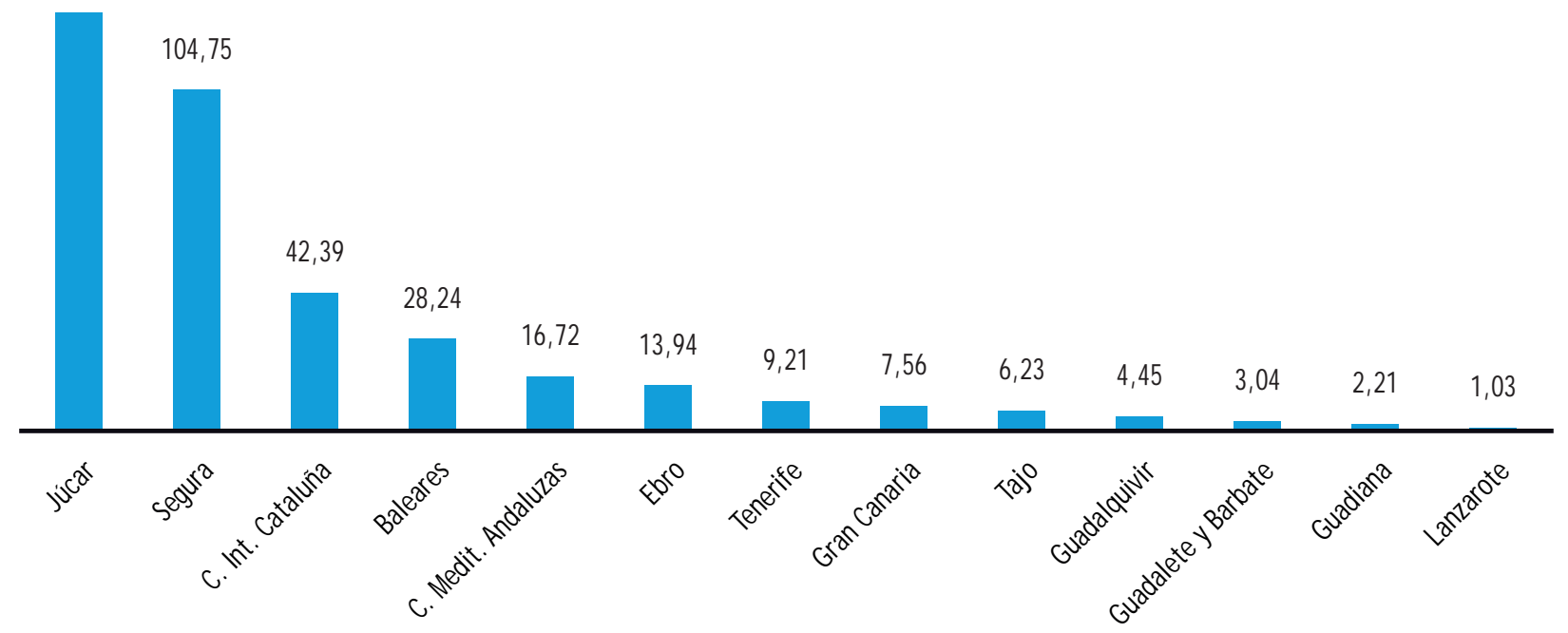

Fuente: CEDEX, 2008.

La práctica de la depuración de aguas residuales en España no ha dejado de crecer en las últimas décadas, y desde la década de los 70 se trata de una tecnología que se ha extendido a todos los núcleos poblacionales. Solo en el periodo 2000-2005 el volumen de agua reutilizada aumentó un 69\%10, evidencia de que la sociedad cada día es más consciente del esfuerzo que debe hacer por regular sus vertidos, a la par que se encuentran cada día más fines para las aguas regeneradas.

\section{LA IMPORTANCIA DEL RIEGO PARA LA ACTIVIDAD AGRARIA}

La agricultura es una gran consumidora de agua. Con el riego se incrementa el aprovechamiento potencial de los suelos y se facilita que las plantas utilicen plenamente los demás factores de producción, mejorando así sus rendimientos. Los regadíos se han vuelto un factor esencial para las economías rurales y con ellos se ayuda a la supervivencia de gran parte de la población mundial.

Al final del siglo XX, la agricultura empleaba por término medio el $70 \%$ de toda el agua utilizada en el mundo. La FAO estima que el agua destinada al riego aumentará aún un 14\% para 2030, sin embargo, no puede incrementarse al ritmo insostenible que se ha dado en las décadas finales del siglo XX.

En el caso de España la transformación de secanos en regadío ha sido durante muchos años la gran esperanza de la agricultura española. Las políticas hidrológicas del siglo XX impulsaron la financiación y la ejecución de grandes obras públicas, pasándose del millón de hectáreas tradicionalmente regadas en España a los 3,5-3,7 millones de hectáreas actuales.

Esta intensificación fue mayor desde 1986, cuando España se incorpora a la Unión Europea. La modernización de las estructuras agrarias en general y del riego en particular, incrementó notablemente la capacidad de producción, facilitando una gran

10 INE, 2008. diversificación de cultivos, en la que el clima mediterráneo es un aliado clave. Las cifras oficiales hablan de que entre 1986 y 2009 se produjo un aumento de la superficie regada cercano a las setecientas mil hectáreas, es decir un crecimiento del 22\%11.

Según afirma el Ministerio de Agricultura, el regadío es una pieza fundamental del sistema agroalimentario español, ya que aporta más del cincuenta por ciento de la producción final agraria, a pesar de que las tierras regadas solo ocupan el 13\% de la superficie agrícola útil. En término medio una hectárea de regadío produce unas seis veces lo que una hectárea de secano, y genera una renta cuatro veces superior ${ }^{12}$.

Pero el regadío también presenta grandes contradicciones. Esta actividad exige disponer de costosas infraestructuras que deben ser renovadas y requieren continuas inversiones para su mantenimiento. Por otra parte, estas infraestructuras han hecho del riego el gran consumidor de agua (aproximadamente el sesenta y ocho por ciento del consumo total), generando polémica y recelos entre los diferentes grupos sociales, ya que surge una gran competencia por un recurso natural realmente escaso en nuestras latitudes y al que la industria, las actividades turísticas y de ocio, la construcción, etc., también quieren acceder.

El regadío español consume $24.500 \mathrm{Hm}^{3}$ de agua al año, de las que dos terceras partes proceden de aguas superficiales y una tercera parte de aguas subterráneas. Otras fuentes menos convencionales, como las aguas regeneradas o las aguas desaladas, son todavía poco frecuentes. Dado que la presión del regadío sobre el consumo de recursos hídricos ya es excesiva, sobre todo en parte de la España central y las zonas mediterráneas, donde más desequilibrio hay entre oferta y demanda de agua, se hace patente que deban encontrarse alternativas a las fuentes tradicionales si se pretende mantener estos niveles de consumo.

11 FNCA, 2016.

12 MAGRAMA, 2016 
En cualquier caso, no está de más recordar que las mejoras tecnológicas que se están generalizando en los regadíos, permiten regar un mayor número de hectáreas con mucha menos cantidad de agua. Es el caso del riego por goteo, que se está extendiendo de manera mayoritaria, y en la mayoría de los casos permite sustituir con gran eficiencia a los sistemas de gravedad e inundación, que son modalidades de riego que requieren de volúmenes mucho mayores de agua.

El reconocimiento social que siempre ha tenido el regadío ha venido ligado a las ventajas del desarrollo económico que ha propiciado. Pero tras un crecimiento descontrolado del número de pozos irregulares y la expansión del riego en zonas con un fuerte déficit de agua, se constata un aumento de la contaminación difusa por nitratos y a la par una disminución en la apreciación social de la población sobre esta modalidad de agricultura. En este momento las administraciones y regantes deben propiciar una nueva cultura del agua. Los objetivos ambientales tan reseñados por la DMA, o Directiva Marco del Agua (2000/60/CE), implican una estricta regulación de los nuevos regadíos, la reducción del consumo de agua y una mejora evidente de la eficiencia de las infraestructuras y de los sistemas de riego.

\section{Posibilidades que ofrecen las aguas Regeneradas EN AGRICULTURA}

Si durante años las necesidades de agua en la agricultura se cubrían plenamente con aguas subterráneas y superficiales, en la actualidad esto no es siempre posible. La disponibilidad de aguas subterráneas hoy por hoy es mucho menor, debido a la sobreexplotación continuada de muchos acuíferos ${ }^{13}$. Tampoco es siempre posible conseguirla por almacenamiento, por lo que surge la necesidad de buscar nuevas alternativas.

A lo anterior se añade otro problema, y es la mala calidad de los recursos tradicionales, ya que las aguas están salinizadas o contaminadas por fertilizantes y otros productos químicos. La posibilidad de utilizar el agua depurada como parte del ciclo hidrológico es la evidencia de la continua adaptación que realiza el ser humano con el medio ambiente.

Encontramos antecedentes de aplicación de aguas residuales a terrenos agrícolas desde la antigüedad, aprovechando su valor fertilizante (ya lo hacían griegos y romanos) o como estrategia para la eliminación de contaminantes antes del vertido a los ríos (referencias hechas en China, Inglaterra o Alemania desde el $\mathrm{s}$. $X V I)$. No obstante, es durante el siglo XX y en el presente cuando más se ha avanzado en este sistema alternativo de depuración. Básicamente los obj etivos han sido regular los recursos hídricos en zonas deficitarias, a la vez que favorecer la resolución de ciertos problemas medioambientales, como ir creando barreras contra la intrusión marina.

En agricultura el problema de escasez de agua viene parejo al de la pérdida de la calidad del suelo. La escasez del agua conlleva la salinización de los terrenos y puede generar desertificación a largo plazo. La falta de precipitaciones disminuye los lavados naturales incrementando la concentración de sales en el

13 Ibáñez et al. 2008. Van Camp et al. 2010. suelo y afectando al rendimiento de los cultivos. Por su parte, los cultivos y su modelo de gestión también tienen sus efectos sobre el suelo: afectan a sus propiedades físicas y químicas, al contenido original de materia orgánica, modifican la estructura, estabilidad, capacidad de amortiguación y retención de agua, la actividad biológica y el intercambio de nutrientes.

El uso de agua regenerada para el riego de los cultivos puede tener un efecto directo en la fertilidad y productividad del suelo, en principio se prevé un impacto positivo, al ser estas aguas ricas en nutrientes y materia orgánica. Sin embargo, un riego prolongado con aguas regeneradas podría generar determinados problemas ambientales, lo que obliga a aplicar un estricto manejo de este tipo de aguas y frecuentes controles para evitar efectos nocivos.

Entre todos los efectos directos y beneficiosos de utilizar el agua depurada para el riego, hay que mencionar la reducción del uso de fertilizantes y el incremento de la productividad agraria en las tierras áridas propias de zonas de baja pluviometría. Por ello se dice que el uso de aguas depuradas en la agricultura es una opción de futuro al incrementar la viabilidad técnica y económica de los cultivos en muchas zonas donde la agricultura es una actividad principal pero que sin embargo dispone de recursos hidrológicos escasos ${ }^{14}$

Por otro lado, sigue siendo preciso superar las dificultades de su aceptación social, para lo que es imprescindible una gestión adecuada ${ }^{15}$. Los inconvenientes se centran en dos aspectos, por una parte en el coste, no solo del recurso en sí sino de toda la infraestructura que se requiere para poder ser llevado a cabo, y por otra la respuesta del cultivo, ya que todas las especies no se adaptan por igual a este suministro. Por ejemplo, se analizan los efectos de utilizar las aguas recicladas en cultivos leñosos ${ }^{16}$ y en cultivos hortícolas ${ }^{17}$.

Si bien en la práctica se constata que el uso de aguas depuradas es limitado en agricultura básicamente por el precio ${ }^{18}$, el garantizar unos determinados suministros hidrológicos también tiene beneficios medioambientales ${ }^{19}$. Otros estudios no alaban las bondades del agua regenerada sino que remarcan más sus inconvenientes, especialmente nombran su elevado precio (debido a su coste de obtención y transporte), el que necesiten ser mezcladas con otras aguas (algo que dificulta su gestión) y sobre todo inciden en los riesgos en cuanto a la posible toxicidad, sobre todo de algunos elementos químicos.

\section{Datos Sobre la aplicación del agua Regenerada EN AGRICULTURA}

Según información publicada por el Ministerio de Agricultu$\mathrm{ra}^{20}$, el principal destino del agua depurada es la actividad agraria

14 Melián, 2005. Navarro Caballero, 2010. Nicolás et al. 2011

15 Candela et al. 2007; Carr et al. 2011.

16 Alcón et al. 2012a.

17 López-Cuquejo, 2001

18 Montesinos, 2004.

19 Alcón et al. 2012b

20 Puig, 2012. 
(sobre el 71\%, mientras que el uso menos representativo es el urbano, al que se dirigiría solo el $4 \%$ del volumen de agua regenerada. Las previsiones indican que el principal incremento será para un uso ambiental (el que se estima consumirá del 17,7\% en 2006 al 21,9\%en el año 2021), el industrial (que pasará del 0,3\%al 12\% en ese mismo periodo) y el urbano (que pasará del 4\%al 9,1\%. En esa evolución se cree que proporcionalmente mermarán las cantidades destinadas a agricultura y actividades de ocio recreativo.

Según el informe CEDEX ${ }^{21}$ de un volumen de aguas regeneradas anual de $261 \mathrm{Hm}^{3} /$ año, el $70 \%$ fue destinado a actividades agrarias. Las Comunidades Autónomas que mayores volúmenes reutilizan para el riego agrícola son: Valencia $\left(113,5 \mathrm{Hm}^{3} /\right.$ año) y Murcia $\left(83,5 \mathrm{Hm}^{3} /\right.$ año). Solo estas dos Comunidades Autónomas acaparan el $75,41 \%$ de las aguas reutilizadas para un fin agrario, o lo que es igual, el 53,53\% del total del caudal regenerado en España el año analizado.

Como es lógico las Comunidades Autónomas con mayor déficit de agua y que a su vez dependen de una agricultura intensiva y muy productiva, son las que más invierten en mecanismos para tener fuentes alternativas de agua que complementen a las tradicionales, como es el caso de las aguas depuradas.

\section{Ventaj as E INCONVENIENTES del USO del agua REGENERADA EN AGRICULTURA}

Con la reutilización de aguas residuales que han sido depuradas, no solo se ayuda a paliar los problemas de escasez de agua en las zonas áridas y semiáridas, también se resuelve un problema medioambiental. Ocurre con frecuencia que el agua residual tratada resulta tener una mejor calidad que el agua original de la zona, adecuándose de mejor manera a su uso en riego ${ }^{22}$. Esto se observa al comparar la analítica de un agua depurada con la de agua de pozo. En general los parámetros claves están controlados en ambos, e indican que las dos son aptas para el riego, sobre todo si nos referimos a salinidad, elementos potencialmente fitotóxicos o bacteriológicos. Pero la realidad es que en zonas con acuíferos sobreexplotados los niveles de boro y sodio son menores si se utiliza un agua que ha sido depurada. Se podría decir, por lo tanto, que la depuración mejora la calidad de unos recursos hidrológicos que están naturalmente deteriorados.

Además, en el caso de que se emplee en el riego de los cultivos un agua regenerada que contenga un determinado nivel de nitratos, esto podrá suponer un ahorro en el posterior uso de fertilizantes. Se trata de aprovechar dichas aguas de modo que se reduzcan los inputs agrícolas, por lo que se producirá un efecto medioambiental positivo (menor aporte de nitratos, menor por lo tanto concentración de nitratos en el agua que percola o de escorrentía y menos riesgo de contaminación de los acuíferos) y un efecto económico sobre la renta del agricultor (al reducirse el coste de los abonos, que suele ser uno de los principales insumos de los cultivos)

21 CEDEX, 2008.

22 López-Cuquejo, 2001.
Los diversos estudios realizados sobre la reutilización del agua depurada en la agricultura mencionan numerosas ventajas de este uso, siendo algunas de las principales las siguientes:

- Se puede tener una mayor garantía en el suministro, el cual puede ser más constante y seguro, ya que será posible disponer del mismo incluso en los periodos de sequía.

- Representa un aporte continuo de nutrientes para las plantas.

- Disminuye los gastos de fertilización como consecuencia del aporte de micronutrientes en el agua residual (básicamente nitrógeno, fósforo y potasio).

- Contribuye a la conservación de recursos hídricos, permitiendo reservar el agua original para otros fines y favorecer el equilibrio ecológico.

- Aunque no ocurre con el resto de alternativas de agua con las que se podría comparar, generalmente el agua regenerada puede representar un coste económico menor al de otras fuentes convencionales.

- Aplicadas de forma correcta, son aguas que contribuyen a la regeneración de suelos ubicados en zonas de baja pluviometría, que en el caso español podrían ser toda la cuenca mediterránea, y garantiza la continuidad de una actividad tan importante como la agraria.

En cuanto a sus inconvenientes, reticencias o desventajas por las que estas aguas no se aplican de manera más mayoritaria, destacan:

- Los agricultores han de dar su apoyo explícito para que puedan ser usadas. Su aceptación dependerá por lo tanto del impacto neto en la renta de las explotaciones y las opciones que les ofrezca el resto de alternativas hidrológicas.

- Uno de los principales miedos son los posibles efectos sobre la salud debido a su contenido microbiológico. Surgen dudas sobre la posible transmisión de enfermedades infecto-contagiosas (bacterias y virus).

- Exigen unas infraestructuras convenientemente diseñadas para que sea factible el tránsito del agua desde las EDAR a las parcelas de riego. Este transporte se convierte con frecuencia en uno de los más difíciles de gestionar y que más encarece el proceso.

- También generan dudas sobre su capacidad fertilizante. Una carga excesiva de N, P y K puede provocar efectos nocivos sobre el terreno, las plantas y sobre el resto de aguas subterráneas.

- Su uso requiere de una evaluación previa de diversos parámetros, que hay que saber gestionar: sólidos en suspensión, pH, conductividad, etc. Por ejemplo, si la materia orgánica que tiene el agua es en principio biodegradable, pero no dispone de suficiente oxígeno, no se degradará adecuadamente y dará lugar a condiciones sépticas. Por su parte, una excesiva conductividad por exceso de salinidad origina problemas de permeabilidad en el suelo, y la concentración de ciertos iones $\mathrm{Na}, \mathrm{Ca}, \mathrm{Mg}, \mathrm{Cl}$, o B provocan diversos daños a los cultivos. El pH del agua, por su parte, puede afectar a la solubilidad de los metales y alterar el equilibrio del suelo.

- Puede darse la presencia de metales pesados (como cadmio, mercurio o zinc) que tienen claros efectos tóxicos para los cultivos y la salud. 
- La simple presencia de sólidos en suspensión, lo que se percibe como turbidez, provoca deterioros en los sistemas de riego localizado, por obturaciones. Además, se pueden formar pequeños depósitos de lodos que generen en el suelo condiciones anaeróbicas.

\section{Requerimientos para la utilización del agua REGENERADA EN AGRICULTURA}

Si la intención es que una zona agraria emplee el agua regenerada como fuente de suministro para el riego, hay una serie de factores que deben ser tenidos en cuenta.

Lo primero es que las aguas residuales depuradas deben cumplir las normas de calidad físico-química que junto a otras características sanitarias o microbiológicas vienen definidas en la propia legislación, actualmente el real decreto 1620/2007. Dichas condiciones de calidad están diferenciadas según los usos a los que se destina el agua, estando el uso agrario en el segundo lugar en el nivel de exigencias. Los parámetros que se controlan son: la presencia o no de nematodos intestinales y de Escherichia coli en el agua, el grado de turbidez, los sólidos en suspensión y la cantidad de nitrógeno y fósforo total. Así mismo, y según el uso, procede ralizar un control de Legionella o Salmonella.

Otro de los requisitos es disponer de la infraestructura necesaria tanto para el tratamiento que necesitan las aguas para ser regeneradas, como para el transporte e intercambio de las mismas y poder con ello reutilizarlas. Se necesitarán, entre otras, conducciones, instalaciones de bombeo, almacenaje, etc., esto en su conjunto supone una gran inversión en elementos inmovilizados que se deben poder amortizar. Sin duda, el lugar donde se ubique la EDAR y cómo se defina el plan de uso, deben ser bien planificados para evitar que los costes finales hagan insostenible el proyecto.

El utilizar agua depurada pasa por garantizar tanto la conservación de la fertilidad del suelo, es decir sus características orgánicas e hidrogeológicas, como la necesidad de obtener producciones con las máximas garantías higiénicas y sanitarias, y es por ello que su manejo y posterior aplicación debe realizarse de modo controlado.

También influyen las características físico-químicas y edáficas del suelo. Por otra parte, serán muy relevantes las características del sistema de riego (si es por gravedad, localizado, etc.). En principio, y tal como se indica en la guía para aplicar correctamente el real decreto 1620/2007, el riego localizado es el método más adecuado para aplicar este tipo de aguas, ya que con otros como el riego por inundación no se podría evitar que se extiendan por otra superficie donde no son útiles. Hay que minimizar el uso de elementos que aumenten la evaporación, escorrentía o infiltración (por ejemplo, los reguladores de presión, goteros autocompensantes, etc.). El riego debe de estar automatizado para evitar el encharcamiento y la escorrentía. Finalmente es muy importante que las personas que manipulan de forma directa este tipo de suministro tomen las precauciones pertinentes para evitar posibles riesgos sanitarios.
En este mismo sentido otras buenas prácticas indican que hay que señalizar debidamente las tuberías para que en caso de avería puedan ser pronto localizadas. Asimismo, se debe evitar regar con agua regenerada a menos de dos semanas de la recolección para que el posible contacto del agua con los frutos no repercuta en la calidad final y comercial de la cosecha.

Respecto al tipo de cultivo es muy limitante porque condiciona la frecuencia del riego y la cantidad de agua que debe ser empleada. Cada especie vegetal muestra una tolerancia distinta al contenido en sales y asimila de forma desigual por ejemplo el nitrógeno o el fósforo (que son dos nutrientes muy frecuentes en las aguas regeneradas). Igualmente va a ser muy importante conocer el valor económico que pueda tener la producción, ya que determinará una elección u otra del suministro hídrico.

En la planificación de una infraestructura hidrológica mediante el suministro de agua depurada, otra de las principales dificultades es poder equilibrar las demandas potenciales con los volúmenes reales. Por la parte de la demanda para los cultivos, esta suele ser muy estacional, y si está altamente concentrada quizás no sea posible satisfacerla.

Finalmente, la viabilidad de un proyecto para la reutilización de las aguas residuales pasa por obtener el apoyo de los agricultores, y esto significa reconocer su innata resistencia a los cambios y su reticencia a la sustitución de los recursos tradicionales por otros nuevos, que en este caso sería utilizar como suministro de agua de riego un agua regenerada. En este sentido se hace necesaria una correcta información inicial y a ser posible una formación práctica a los futuros usuarios. Sin duda conocer cómo debe hacerse un buen manejo de las mismas es lo que hará que se consolide el uso del agua regenerada en la agricultura, y con ello pasar de ser un recurso minoritario y no convencional, a convertirse en uno estratégico y la solución para la agricultura de regadío en muchas comarcas agrarias españolas.

\section{Posibles problemas derivados del uso de agua DEPURADA EN EL RIEGO}

Los principales aspectos a considerar en la evaluación agronómica del uso de este tipo de aguas en el riego serían: salinidad, macronutrientes, micronutrientes, metales pesados, pH, sólidos en suspensión, cloro residual, etc. Por una parte, si tienen una excesiva proporción de iones $\mathrm{Na}, \mathrm{Ca}, \mathrm{Mg}, \mathrm{Cl} \circ \mathrm{B}$, se pueden producir daños a los cultivos y provocar problemas de permeabilidad en el suelo. El contenido de minerales como nitrógeno, fósforo, potasio y ciertos microelementos, reduce las necesidades de fertilización ${ }^{23}$ pero todos ellos son igual de buenos en una proporción correcta como malos si su concentración es excesiva.

Por su parte, los sólidos en suspensión que se originan por la precipitación de elementos químicos, presencia de algas, etc., pueden obstruir los goteros y otro tipo de emisores del riego localizado. Los sólidos en suspensión también taponan los poros del suelo, reduciendo la aireación y las vías por las que penetra

23 Asano et al. 1991. 
Gráfico 2. Recomendaciones de utilización de aguas depuradas para uso agrícola

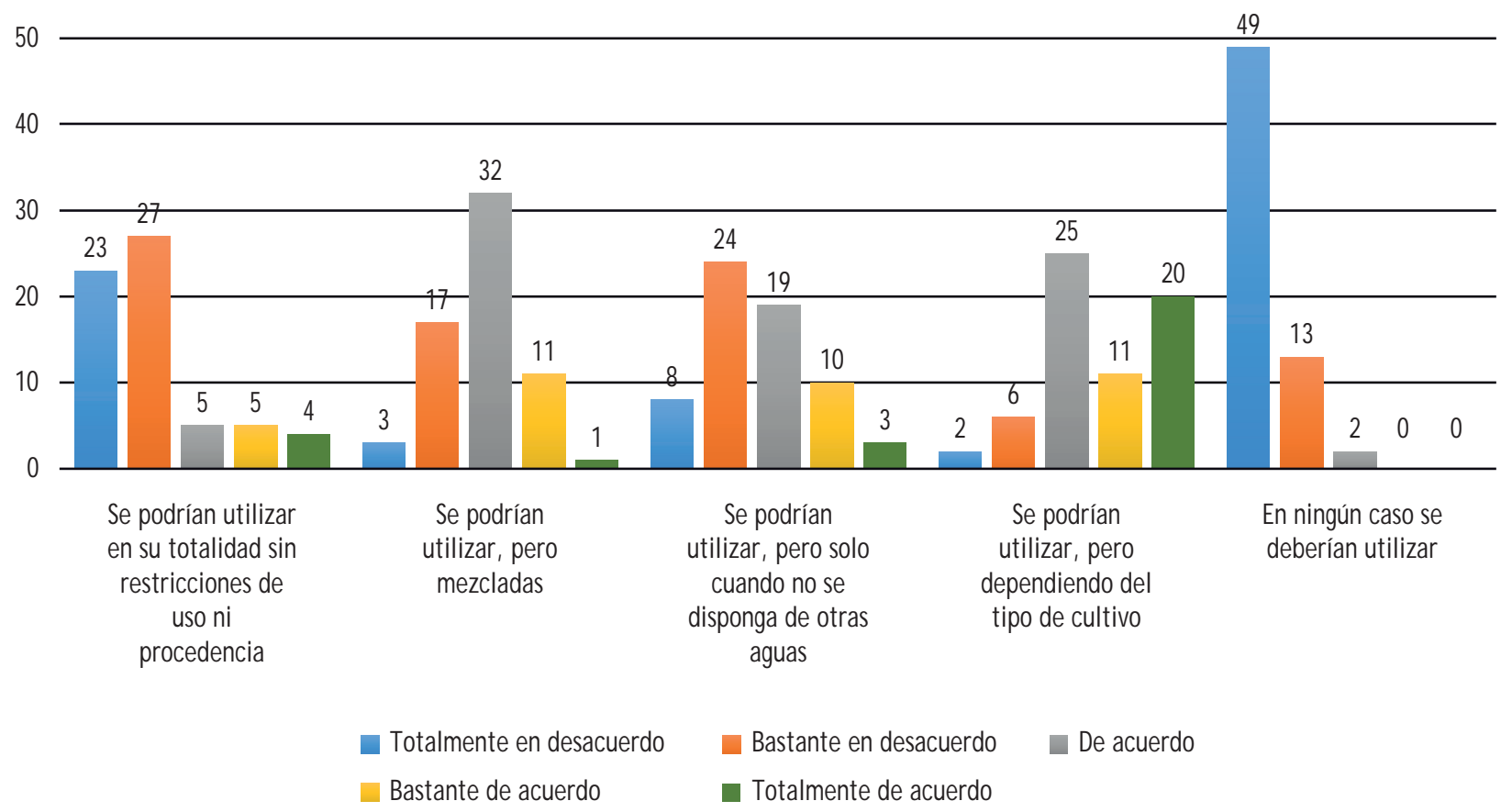

Fuente: Costa y Melián, 2015.

el agua. Una buena alternativa a estos problemas es someter el agua residual a un tratamiento secundario, lo que reduciría considerablemente los sólidos en suspensión y, además limpiar con cierta regularidad las tuberías, utilizar filtros y cloro para prevenir la obturación de los goteros.

\section{ACEPTACIÓn DEL USO de AGUA DEPURAda EN AGRICULTURA}

De forma global los posibles riesgos de seguridad alimentaria son los que más utilizan los detractores del uso de agua regenerada en la agricultura, haciendo que haya una percepción de alguna manera negativa, percepción que dificulta la expansión de esta modalidad de agua. Un estudio cualitativo ${ }^{24}$ con el que se consulta a expertos para sondear el grado de aceptación del uso del agua depurada en la agricultura, muestra las posibilidades de aplicación en el sureste español. Este estudio se fundamenta en la experiencia y opinión de: agricultores regantes, que son los usuarios directos del agua, técnicos y otros responsables de las Comunidades de Regantes y J uzgados Privativos del Agua, gestores de empresas relacionadas con el agua, investigadores, profesores de universidad y funcionarios vinculados al ámbito hidrológico. Se trataba, por lo tanto, de sondear la aceptación por parte de los agentes que podrían utilizar directamente este tipo de aguas o bien intervenir en la difusión de esta tecnología.

La encuesta, realizada en 2013, recopiló información aportada por 65 expertos. Sus opiniones fueron clasificadas mediante una escala Likert tabulada de 1 a 5. Los aspectos analizados responden a dos preguntas claves: perspectivas de uso de aguas

24 Costa, 2015. depuradas en agricultura y principales inconvenientes del uso del agua depurada en agricultura.

En lo relativo a las perspectivas se plantean cinco opciones desde la más amplia (utilizar este tipo de agua sin restricciones) hasta la más restrictiva (no utilizar en ningún caso) -gráfico 2-. Los resultados indican que no hay un rechazo explícito a esta fuente de agua y tampoco se condiciona su uso al mero hecho de no disponer de otro tipo de agua, sino que ven asumible su utilización. Sin embargo, los entrevistados inciden en que se apliquen sobre todo mezcladas con otras aguas y que su uso se condicione al tipo de cultivo al que se refieran.

En lo relativo a los inconvenientes que ven en las mismas, se señala el riesgo de toxicidad (que es el aspecto en el que hay un mayor consenso) pero también se apuntan los problemas que podrían causar en las instalaciones de riego por goteo, la necesidad de bombearlas desde depuradoras (con el consiguiente incremento de coste) y la necesidad de almacenamiento en balsas por la discontinuidad del caudal disponible -gráfico 3-.

\section{UTILIZACIÓN DE LOS LODOS DE LAS DEPURADORAS EN AGRICULTURA}

Además del agua regenerada, las depuradoras producen lodos de depuración, un subproducto derivado de los propios tratamientos de las aguas residuales. Estos lodos son ricos en materia orgánica y nutrientes, por lo que pueden ser utilizados como enmienda orgánica en suelos pobres.

El uso de lodos de depuración en la actividad agraria se regula a través del real decreto 1310/1990, en el que se establece los requisitos y cualidades físico-químicas que deben cumplir los lodos para su utilización. Los que incumplan los límites estable- 


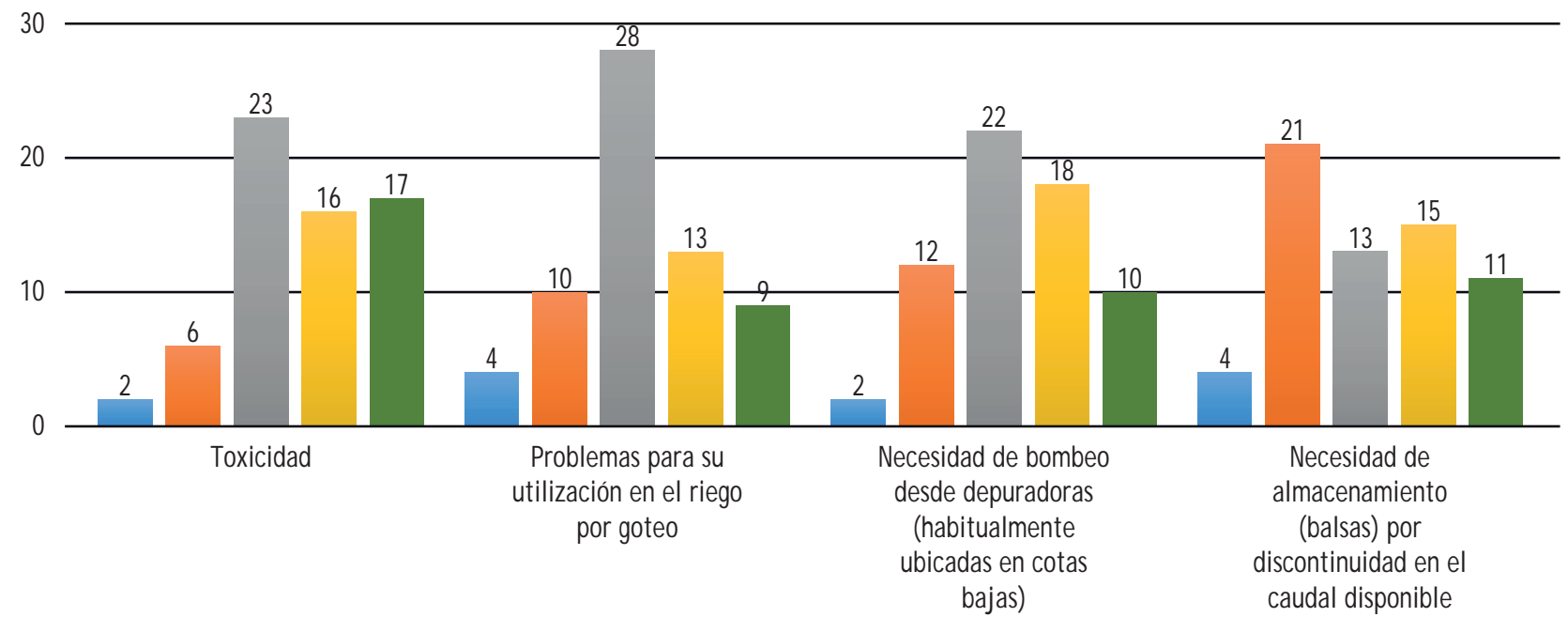

$\begin{array}{ll}\text { Totalmente en desacuerdo } & \text { Bastante en desacuerdo } \square \text { De acuerdo } \\ \text { Bastante de acuerdo } & \text { Totalmente de acuerdo }\end{array}$

Fuente: Costa y Melián, 2015.

cidos también se utilizan, pero en este caso se les da un secado térmico y se destinan a usos energéticos.

Según EPSAR ${ }^{25} 2015$, organismo que sirve de coordinación entre las distintas EDAR de la Comunidad Valenciana, en esta región se produjeron en 2014 un total de 360.000 t de lodo, una cifra que en el año 2005 llegó a superar las 500.000 t. Dichos lodos se distribuyeron para un uso agrario (un 83\% del total se aplicó directamente al terreno y un $5 \%$ previamente se compostó y luego fue aplicado), distribuyéndose por una extensión total de 14.139 ha, a las que de media se aplicó 21,1 t de materia húmeda por hectárea. El resto, el otro 12\% del total, se destinó a los hornos de cementera.

Los lodos, por lo tanto, hay que valorarlos como un resultado más del proceso de regeneración de las aguas residuales y no hay que verlos como un problema, sino como una valiosa fuente de materia orgánica y nutrientes para el campo.

\section{Datos prácticos SObre el uso del agua REGENERADA EN AGRICULTURA}

Para finalizar esta parte sobre el uso del agua regenerada en agricultura se comentan algunas experiencias prácticas con las que se trata de conocer desde el punto de vista científico-técnico los efectos de este uso. Algunas investigaciones ${ }^{26}$ se han centrado en hacer un seguimiento sobre los efectos en el suelo agrario a corto y medio plazo. Asi, tras un ensayo de cuatro años en tres

25 EPSAR, 2015.

26 Morugán-Coronado, 2011. zonas de la provincia de Alicante, muestra que no existen diferencias importantes entre los suelos regados con aguas residuales depuradas y aquellos regados con aguas convencionales, ya que solo se encontraron ligeras variaciones en algunas de las propiedades del suelo estudiadas (como una ligera modificación puntual de la conductividad eléctrica) pero no había cambios respecto a las actividades enzimáticas.

En cuanto a otros parámetros relacionados con la conservación, degradación, salinidad, fertilidad y contaminación del sue10, así como sobre la productividad de un cultivo sometido a un riego con agua residual depurada, la citada investigación indicó que se encontraron aumentos puntuales de sodio asimilado y un aumento ligero de fósforo, mientras que no se apreció ningún cambio notable en el pH, ni en el calcio, nitrógeno, magnesio, cinc, cobre, manganeso, cadmio, plomo, cromo... Por lo tanto, no se percibió ninguna variación significativa en las propiedades del suelo. En las condiciones estudiadas se concluye que el uso de las aguas residuales para riego no altera las propiedades del suelo.

En otros trabaj $0 S^{27}$ se revisan las distintas experiencias realizadas en campo. Así, en el Campo de Dalías (Almería), según señalaba en la década de los 90 el Plan de Ordenación del Territorio de la Comarca del Poniente Almeriense, gran parte de los volúmenes de agua depurada se destinarán a la agricultura intensiva, para campos de golf una vez pasada la campaña agrícola o para la infiltración en el terreno. El volumen inicial previsto para depurar y reutilizarse era de $10 \mathrm{Hm}^{3}$, no siendo cifras significativas respecto al consumo total y el déficit hídrico que tiene la comarca, pero

27 López-Cuquejo, 2001 
dado que era el inicio de esta tecnología se puede clasificar como una cifra interesante.

El estudio habla de destinar estas aguas a los cultivos hortícolas en invernadero (hortalizas y flores), atribuyendo a cada agricultor una dotación de $7.000 \mathrm{~m}^{3} /$ ha y año, cantidad que podía incrementarse, a la par que lo haría su precio medio, con la intención de que los agricultores contuvieran el gasto. Los efectos obtenidos son bastante buenos, según menciona el autor, quien los resume diciendo que gracias al agua regenerada se ha conseguido que:

- Sea posible una alternativa de cultivos, ya que con los pozos originales de agua, muy salinizados, el tomate era casi la única verdura que podían cultivar.

- Los rendimientos de las cosechas han subido hasta duplicarse en el tomate, desde que se utilizan aguas depuradas, las cuales tienen mejor calidad que muchas de pozos tradicionales. Especialmente tienen mucha menos concentración de boro que las aguas naturales, y al venir con cierta riqueza de nitrógeno y fósforo suponen además un ahorro en fertilizantes.

- Los agricultores pueden programar sus riegos. Al ser la demanda conocida y controlada por la Comunidad de Regantes, agiliza notablemente la gestión y mejora la calidad del trabajo del agricultor.

- Finalmente, el agua ahora llega para todos los usuarios, evitando los conflictos surgidos en una zona muy árida donde el agua es un factor muy limitante y apenas hay venta de caudales de pozos particulares.

También señala los inconvenientes detectados, que son los ya mencionados de forma genérica, y no se aprecian grandes problemas, siendo quizás la presencia de sólidos en suspensión lo que requiere de una mayor atención en la gestión.

Finalmente, y dado que junto a las reticencias por posibles riesgos sanitarios los agricultores parecen condicionar su uso al precio que se ofrezcan las aguas regeneradas, hay cifras -relativamente recientes- que informan de este dato. Por ejemplo, las estadísticas oficiales del INE señalan para 2013 como coste unitario del saneamiento de aguas un dato global a nivel nacional de 0,70 euros $/ \mathrm{m}^{3}$. Se trata de una cifra muy elevada si se compara con los precios medios que tiene el agua convencional de riego, los cuales en ocasiones son realmente simbólicos. Otras fuentes citan ${ }^{28}$ el coste para el tratamiento de aguas residuales (incluyendo tratamiento terciario) que oscila entre 0,6 y 0,8 eu$\mathrm{ros} / \mathrm{m}^{3}$, aunque especifica la gran variabilidad existente según los distintos tipos de tratamientos y las condiciones en que estos se dan. Este autor plantea una cuestión trascendental: la necesidad de repartir los costes de la regeneración y gestión del agua entre la totalidad del consumo. Parece una reflexión lógica ya que los efectos positivos que con ellas se consiguen son extrapolables a toda la sociedad.

\footnotetext{
28 Melgarej0, 2009
}

\section{Aspectos medioambientales del agua regenerada}

J unto con la agraria, la otra vertiente que hay que valorar del agua regenerada, es su utilidad para el medio ambiente. El mero hecho de que las aguas sean depuradas antes de ser vertidas de nuevo a la naturaleza ya es una cuestión muy relevante. El que de esa manera las aguas puedan ser reutilizadas para otros fines tiene a su vez grandes ventajas.

En los apartados anteriores ya se ha comentado cómo las aguas regeneradas son una alternativa o complemento al resto de suministros tradicionales. Dada la fuerte demanda agraria y urbana que existe sobre los recursos hídricos, el poder contar con una nueva fuente de agua permite sobre todo reducir la sobreexplotación de acuíferos. Este hecho es muy necesario, sobre todo si los acuíferos tienen dificultades para regenerarse naturalmente, 0 bien hay problemas de intrusión de aguas saladas, algo muy frecuente en zonas cercanas a la costa. Al utilizar aguas regeneradas se puede racionalizar la extracción de estas aguas subterráneas, dejando margen para que se recuperen de manera natural, algo ya de por sí difícil en las zonas de escasa precipitación. Sin duda, este podría ser su primer gran beneficio medioambiental, pero tiene otros.

Algunos de los beneficios ambientales de utilizar aguas regeneradas se desprenden de los propios usos que tienen. Por ejemplo, usadas para el riego con frecuencia permiten reducir la carga de fertilizantes en los cultivos, algo muy beneficioso para el medio ambiente: se consume menos productos químicos con el consiguiente ahorro energético que eso conlleva y se evita la acumulación excesiva de químicos sintéticos en los suelos y capas freáticas, una reducción del nivel de contaminación que redunda positivamente en toda la cadena trófica y en la salud de los seres vivos.

Los usos del agua regenerada con un fin ambiental están contemplados en el real decreto 1620/2007, donde también se especifican los requisitos en cuanto a los parámetros de calidad que se exigen para cada uno de ellos. En este reglamento se atribuye al agua regenerada los siguientes usos ambientales:

- Recarga indirecta de acuíferos, es decir aquella que se da cuando el agua entra en los mismos percolando a través del terreno.

- Recarga directa de acuíferos, cuando se producen inyecciones de agua específicamente para este fin.

- Riegos de bosques, zonas verdes y en silvicultura.

- Mantenimiento de humedales, contribución a caudales ecológicos mínimos y otros fines ambientales similares.

\section{Aguas Regeneradas Y SU VINCULACIÓN CON LOS HUMEDALES}

En 1975 entró en vigor la Convención de Ramsar sobre los Humedales de Importancia Internacional. En ella se reconoce la importancia de los humedales como prestadores de servicios ecológicos fundamentales, no solo porque son unos estupendos reguladores de los regímenes hídricos, también son una fuente de biodiversidad a nivel de especies y de ecosistemas. Son bienes naturales de gran valor económico, científico, cultural y recreativo, además de desempeñar un papel esencial en la adaptación 
al cambio climático y para la atenuación de sus efectos más negativos.

El papel que realizan los humedales naturales son en su mayoría factibles de realizar por humedales artificiales, llamados también sistemas de lagunaje. Se trata de una serie de estanques 0 lagunas artificiales de agua depurada que en algunos casos llegan a cumplir una doble función. Por una parte, juegan un rol social y económico al actuar como reservorios de agua depurada que se puede usar en regadíos sin comprometer la calidad de los productos obtenidos. Por otra parte, además de facilitar el desarrollo de actividades de educación ambiental y de ocio en la naturaleza pueden tener una función ecológica, al funcionar como un humedal, en el que se ha desarrollado un ecosistema de agua dulce, con un entorno de diferentes hábitats de interés, y en los que es normal la presencia de diversas especies de aves ${ }^{29}$.

\section{LOS FILTROS VERDES}

Los filtros verdes son un sistema natural de depuración de aguas. Cuando se aporta un caudal controlado de agua de mala calidad sobre la superficie de un terreno, donde previamente se ha instalado una masa forestal o cultivo, esta hará de método natural para depurar el agua antes de que se reintegre de nuevo en la naturaleza. El agua se aplica al terreno mediante riego a manta u otro sistema de inundación. Con este sistema, a la vez que se favorece el crecimiento de especies vegetales, generalmente arbóreas y maderables, se produce una recarga artificial de los acuíferos, obteniéndose, por lo tanto, un beneficio económico y otro ambiental.

La depuración se realiza mediante la acción conjunta del suelo, los microorganismos y las plantas, en un proceso que pasa por una triple acción, ya que hay una filtración física, unos fenómenos químicos al haber intercambios iónicos, precipitación, etc., y procesos biológicos de degradación de la materia orgánica de las capas del terreno afectadas.

Este fenómeno de filtros verdes es lo que se está produciendo cuando se aplica un agua regenerada en el riego de un cultivo, como podría ser el arrozal de humedales tan emblemáticos como la Albufera de Valencia, las marismas de Doñana o el Delta del Ebro. Lo que está ocurriendo es que el propio cultivo del arroz funciona como un filtro verde, permitiendo que el agua vaya depurándose cada vez más según fluye de parcela en parcela.

\section{Datos prácticos sobre el uso del agua regenerada EN EL MEDIO AMBIENTE}

Tal y como se ha realizado antes al hablar del uso del agua regenerada en agricultura, citamos algunas experiencias prácticas sobre la aplicación del agua regenerada con un fin medioambiental.

En un trabajo centrado en la Albufera de Valencia ${ }^{30}$-zona de incalculable valor ambiental que ha sufrido una severa degra-

\footnotetext{
29 Ballesteros, 2012

30 Cifres et al. 2006.
}

dación producto de la presión del desarrollo urbano e industrial del área metropolitana de Valencia-se pone de manifiesto cómo el uso del agua depurada puede ayudar a recuperar el estado ecológico del lago, algo que, por otra parte, viene condicionado por la entrada en vigor de la propia DMA. Las características de la Albufera de Valencia eran a finales de los años 70 ya negativas como consecuencia de entradas excesivas de materia orgánica alóctona y nutrientes inorgánicos, originando un zooplancton reducido comparado con la gran cantidad de fitoplancton, y pobre fauna béntica. Para comenzar a revertir esta situación ha sido necesaria la reducción de las cargas de nutrientes (especial mente el fósforo) mediante actuaciones de saneamiento y depuración, así como asegurar los flujos de agua adecuados. La reutilización de las aguas depuradas en las zonas regables del entorno de La Albufera puede reconducir estos aportes de nutrientes hacia la biomasa del regadío evitando su entrada en el lago y restituyendo su situación al mejorar el estado ecológico del mismo tal y como dicta la DMA.

Otra experiencia ${ }^{31}$ analiza las lagunas de Campotéjar, en Molina de Segura (Murcia), cuyo sistema de depuración mediante lagunaje sustituye a las antiguas depuradoras y convierte estos lagos en auténticos refugios de flora y fauna. Algunas aves de las que nidifican en estos lagos artificiales están amenazadas en el contexto europeo y mundial. Todo esto les convierte en un modelo de sostenibilidad que compatibiliza la depuración del agua y su uso agrícola posterior con la conservación de la naturaleza.

En otro trabaj $0^{32}$ centrado también en la Albufera de Valencia y su Parque Natural estudia los efectos ambientales del riego con aguas residuales depuradas en el cultivo del arroz. La zona se surte de agua de diversas procedencias siendo una de ellas la cercana depuradora de Pinedo. Esta depuradora tiene una capacidad de depuración de 375 millones de litros diarios, siendo la EDAR más grande de la Comunidad Valenciana y una de las mayores de España. En un 50\% sus aguas regeneradas tienen un aprovechamiento agrícola (concretamente para el riego) y el otro $50 \%$ un fin medioambiental, ya que sirve de recarga del lago y zonas húmedas que hay en la Albufera, 0 bien son aguas saneadas que se vierten al mar.

\section{Conclusiones}

Las limitaciones ambientales de ciertos territorios, la escasez hídrica o las sequías plurianuales llevan a ciertas poblaciones a plantearse la utilización de aguas depuradas como fuente hídrica complementaria 0 adicional, sobre todo en aprovechamientos que no requieran la calidad que tiene un agua potable.

La viabilidad de la reutilización dependerá de que sean equilibrados los beneficios aportados respecto a los costes que acarrean. El valor económico del agua que se libera es clave ya que es muy versátil en sus usos, que pueden ser agrarios, urbanos, industriales y medioambientales. Por lo tanto, son una alternativa que evita tener que buscar recursos de un coste mayor. El valor

31 Ballesteros, 2012.

32 Martínez-Cortijo, 2003 
social y medioambiental se logra al impedir que se viertan aguas residuales sin tratar a los sistemas costeros 0 a los acuíferos, lo que a medio y largo plazo está beneficiando tanto a una actividad como la turística como al mantenimiento de los ecosistemas.

El principal uso del agua regenerada, entre todos los posibles, es el agrícola suponiendo un suministro de agua imprescindible sobre todo para las zonas de mayor déficit hídrico. Tienen también el beneficio añadido de que el agua regenerada incorpora nutrientes que son beneficiosos para el suelo y necesarios para los cultivos, por lo que el agricultor obtiene un considerable ahorro en fertilizantes.

Aunque si hay que señalar solo uno, el uso de mayor valor debe ser el medioambiental, donde el agua regenerada juega un papel clave en el mantenimiento de ciertas zonas húmedas. Se trata de contrarrestar las filtraciones salinas marinas o la sobreexplotación a la que se someten en la actualidad a los acuíferos. En el caso concreto de los parajes que forman los humedales, no sería justo valorar su importancia solo por sus actividades económicas (agricultura o turismo) ya que la vida natural que hay en ellos, y que se podría mantener gracias a la aportación de aguas regeneradas, es un intangible de un gran valor para las generaciones presentes y futuras.

\section{Bibliografía}

Aquarec, 2006: Integrated Concepts for Reuse of Upgraded Wastewater. Handbook on feasibility studies for water reuse systems. Aachen, Aquarec (www. aquarec.org).

Alcón, F., Egea, G. y Nortes, P. A. 2012a: "Financial feasibility of implementing regulated and sustained deficit irrigation in almond orchards", en Irrigation Science, 31, 5, 931-941. doi:10.1007/ s00271-012-0369-6.

Alcón, F., Martin-Ortega J., Berbel, J. y de Miguel, M. D. 2012b: "Environmental benefits of reclaimed water: an economic assessment in the context of the Water Framework Directive", en Water Policy, 14, 1, 148-159. DOI: 10.2166/wp. 2011.001.

Asano, T. 1991: "Planning and Implementation of Water Reuse Projects", en Water Science and Technology, 24, 9, 1-10.

Ballesteros Pelegrín, G. A. 2012: "Sostenibilidad social, económica y ambiental en la depuración de agua para uso agrícola y conservación de la naturaleza: las lagunas de Campotéjar (Murcia)", en Papeles de Geografía, 55-56, 11-24.

Ballesteros, G. A., González, C. y Picavet. F. 2008: "Ficha de Información Ramsar de las Lagunas de Campotéjar. Murcia". Consejería de Agricultura de la Región de Murcia, en http:// www. murcianatural. carm.es.

Candela, L., Fabregat, S., J osa, A., Suriol, J., Vigués, N. y Mas, J. 2007: "Assessment of soil and groundwater impacts by treated urban wastewater reuse. A case study: Application in a golf course (Girona, Spain)", en Science of the Total Environment, 374, 1, 26-35. DOI: 10.1016/ j. scitotenv. 2006.12.028.

Carr, G., Potter, R. B. y Nortcliff, S. 2011: "Water reuse for irrigation in J ordan: Perceptions of water quality among farmers", en Agricultural Water Management, 98, 5, 847-854. http://dx. doi. org/ 10.1016/ j. agwat. 2010.12.011.
CEDEX, 2008: Realización de una base de datos sobre los sistemas de reutilización de aguas depuradas en España. Madrid. Centro de Estudios y Experimentación de Obras Públicas. Ministerio de Agricultura, Alimentación y Medio Ambiente.

Cifres, E., Mondría, M., y J uan Ferruses, F. 2006: "Actuaciones del programa AGUA para el desarrollo sostenible de l'Albufera de Valencia", en III Congreso de Ingeniería Civil, Territorio y Medio ambiente: Agua, diversidad y energía. Zaragoza, 25-27 de octubre.

Costa Botella, D. A. 2015: "Estudio de gestión hídrica en la Comarca de la Vega Baja del Segura. Especial incidencia en el uso agrario del agua", tesis doctoral, Universidad Miguel Hernández, Elche.

Costa Botella, D. A. y Melián, A. 2016: "Los retos del uso del agua depurada y desalada en el Sureste español: aplicación a la agricultura", en Navarro Caballero, Teresa M. (dir.): Desafíos del Derecho de Aguas. Variables jurídicas, económicas, ambientales y de Derecho comparado. Aranzadi editorial.

EPSAR, 2015: Entitat de Sanejament d'Aigües. Memoria de Gestión 2014 (Disponible en: http:// www. epsar. gva. es/ sanejament/ quienessomos/ INFORME-DE-GESTION. pdf).

FAO (Food and Agricultural Organization). 2013: Reutilización del agua en la agricultura: iBeneficios para todos? Informe sobre Temas Hídricos, 35. Roma, FAO.

FNCA (Fundación Nueva Cultura del Agua). 2016: Guía nueva cultura agua. Recurso electrónico disponible en: http:// www.fnca.eu/ guia-nueva-cultura-del-agua/

Hernández Sancho, F., De las Fuentes, L. y Urkiaga, A. 2006: Guía para la realización de estudios de viabilidad en proyectos de reutilización de aguas depuradas. Madrid. AQUAREC, MIMAM y Ministerio de Fomento.

Ibáñez, J., Martínez-Valderrama, J. y Puigdefábregas, J. 2008: "Assessing overexploitation in Mediterranean aquifers using system stability condition analysis", en Ecological Modelling, 218, 3-4, 260-266. http:// dx. doi. org/ 10.1016/ j. ecolmodel.2008.07.004

INE. 2008: Estadísticas del agua. Boletín cifras INE. 1/ 2008 (disponible en http:// www. ine.es/ revistas/ cifraine/ 0108. pdf).

Keremane, G. 2010: "Urban wastewater reuse and sustainable development", en Daniels, C. B. (coord.): Adelaide: Water of a City. Adelaide, Wakefield Press, 330-333.

López-Cuquej 0, A. 2001: "Reutilización de aguas depuradas en la provincia de Almería: el caso del aprovechamiento para regadío agrícola en el Bajo Andarax", en Nimbus, 7-8, 123-134.

MAGRAMA, 2016: Ministerio de Agricultura, Alimentación y Medio Ambiente. Información sobre Agua (http://www. magrama.gob.es/ es/agua/ temas/ default. aspx, consulta realizada el 17 de febrero 2016).

Martínez-Cortijo, F. J. 2003: "Estudio agronómico y ambiental del riego con aguas residuales depuradas en el cultivo del arroz. Aplicación a una línea de riego en el Parque Natural de la Albufera (Valencia)", tesis doctoral, Universidad Politécnica de Valencia, Valencia.

Melgarejo, J. 2009: "Efectos ambientales y económicos de la reutilización del agua en España", en Clm. Economía, 15, 245-270.

Melián, A. 2005: "El costo del agua desalada", en X Semana Agrícola de Guardamar, El Agua y los Riegos, Guardamar del Segura (Alicante), 2-10 de noviembre.

Molinos Senante, M., Hernández Sancho, F. y Sala Garrido, R. 2012: "Estado actual y evolución del saneamiento y la depuración de aguas residuales en el contexto nacional e internacional", en Ana- 
les de Geografía, 32, 1, 69-89. http:// dx. doi.org/10.5209/ rev_ AGUC. 2012.v32.n1.39309.

Montesinos, A. 2004: "Estudio de la depuración del agua en España y en la Región de Murcia", proyecto fin de carrera, Universidad Politécnica de Cartagena, Cartagena. PMCid: PMC1615751

Morugán-Coronado, A. 2011: "Efectos de la aplicación de aguas residuales depuradas sobre suelo agrícola", tesis doctoral, Universidad Miguel Hernández, Elche.

Navarro Caballero, T. M. 2010: Reutilización de aguas regeneradas. Aspectos tecnológicos y jurídicos. Murcia, Fundación Instituto Euromediterráneo del Agua.

Nicolás, E., Pedrero, F., Alarcón, J. J ., Mounzer, O., Martínez, V., Nortes, P. A., Alcón, F. J., Egea, G. y De Miguel. M. D. 2011: Estudio de la viabilidad de uso de las aguas regeneradas procedentes de la EDAR de J umilla en la Comunidad de Regantes Miraflores. Murcia, Consejería de Agricultura y Agua Región de Murcia.

Puig Infante, A. 2012: Reutilización de las aguas residuales. Dirección General del Agua-MAGRAMA. Disponible en: http:// www. magrama. gob.es/ es/agua/ temas/ delimitacion-y-restauracion-del-dominiopublico-hidraulico/Reutilizacion_aguas_tcm7-213477. pdf (consulta realizada el 19 de febrero de 2016).

Van Camp, M., Radfar, M. y Walraevens, K. 2010: "Assessment of groundwater storage depletion by overexploitation using simple indicators in an irrigated closed aquifer basin in Iran", en Agricultural Water Management, 97, 11, 1876-1886. http:// dx. doi.org/10.1016/ j.agwat. 2010.02.006 